\title{
Strong compactness, measurability, and the class of supercompact cardinals
}

by

\author{
Arthur W. Apter (New York, NY)
}

\begin{abstract}
We prove two theorems concerning strong compactness, measurability, and the class of supercompact cardinals. We begin by showing, relative to the appropriate hypotheses, that it is consistent non-trivially for every supercompact cardinal to be the limit of (non-supercompact) strongly compact cardinals. We then show, relative to the existence of a non-trivial (proper or improper) class of supercompact cardinals, that it is possible to have a model with the same class of supercompact cardinals in which every measurable cardinal $\delta$ is $2^{\delta}$ strongly compact.
\end{abstract}

1. Introduction and preliminaries. It is well known that the structure of the class of supercompact cardinals can vary quite a bit. The work of Magidor [16] shows that it is consistent, relative to a supercompact cardinal, for the least supercompact cardinal to be the least strongly compact cardinal. The work of Kimchi and Magidor [13] shows that it is consistent, relative to a proper class of supercompact cardinals, for the classes of supercompact and strongly compact cardinals to coincide precisely, except at measurable limit points, where a result of Menas [18] shows that this is impossible. The work of [4] shows that, relative to the existence of a cardinal $\Omega$ which is an inaccessible limit of measurable limits of supercompact cardinals, it is consistent for the classes of supercompact and non-supercompact strongly compact cardinals to have, roughly speaking, any conceivable structure dictated by a ground model function $f: \Omega \rightarrow 2$.

2000 Mathematics Subject Classification: 03E35, 03E55.

Key words and phrases: supercompact cardinal, strongly compact cardinal, nonreflecting stationary set of ordinals.

The author wishes to thank James Cummings for helpful discussions on the proof of Theorem 2, and the referee for numerous comments and suggestions which have been incorporated into this version of the paper. 
The purpose of this paper is to explore additional possibilities for how strong compactness and measurability can interact with the class of supercompact cardinals. Specifically, we prove the following two theorems.

Theorem 1. Suppose $V \vDash \mathrm{ZFC}$ and $\mathfrak{K} \subseteq V$ is so that $\mathfrak{K} \neq \emptyset$ is the (possibly proper) class of supercompact limits of supercompact cardinals. Assume further that if $\mathfrak{K}$ is a set, $V \vDash$ "No cardinal $\lambda>\sup (\mathfrak{K})$ is supercompact". There is then a partial ordering $\mathbb{P} \subseteq V$ with $V^{\mathbb{P}} \vDash$ "ZFC + The only supercompact cardinals are the elements of $\mathfrak{K}+$ Every supercompact cardinal is a limit of (non-supercompact) strongly compact cardinals".

Theorem 2. Suppose $V \vDash \mathrm{ZFC}$ and $\mathfrak{K} \subseteq V$ is so that $\mathfrak{K} \neq \emptyset$ is the (possibly proper) class of supercompact cardinals. There is then a partial ordering $\mathbb{P} \subseteq V$ with $V^{\mathbb{P}} \vDash$ "ZFC + The only supercompact cardinals are the elements of $\mathfrak{K}+$ For every cardinal $\delta, \delta$ is measurable iff $\delta$ is $2^{\delta}$ strongly compact".

To a large extent, the proofs of the above two theorems will rely on Hamkins' work of [10]-[12]. Theorem 1 extends and generalizes Corollary 4 of [4]. Theorem 2 provides instances of models with supercompact cardinals in which every measurable cardinal has a non-trivial degree of strong compactness. Theorem 2 should be contrasted with the models constructed in [7]. In these models, which contain supercompact cardinals, for regular cardinals $\kappa<\lambda, \kappa$ is $\lambda$ strongly compact iff $\kappa$ is $\lambda$ supercompact, except possibly if $\kappa$ is a measurable limit of cardinals $\delta$ which are $\lambda$ supercompact. Note that Theorem 1.1 of [2] provides an example of a universe in which every measurable cardinal has a non-trivial degree of strong compactness, but in which no supercompact cardinals are present.

We take the opportunity now to mention some preliminary material. If $\alpha<\beta$ are ordinals, then $[\alpha, \beta],[\alpha, \beta),(\alpha, \beta]$, and $(\alpha, \beta)$ are as in standard interval notation.

When forcing, $q \geq p$ will mean that $q$ is stronger than $p$. If $\mathbb{P}$ is our partial ordering, $V^{\mathbb{P}}$ and $V[G]$ will be used interchangeably to denote the generic extension when forcing with $\mathbb{P}$. We may, from time to time, confuse terms with the sets they denote and write $x$ when we actually mean $\dot{x}$, especially when $x$ is in the ground model $V$.

The partial ordering $\mathbb{P}$ is $\kappa$-directed closed if for every cardinal $\delta<\kappa$ and every directed set $\left\langle p_{\alpha}: \alpha<\delta\right\rangle$ of elements of $\mathbb{P}$ (where $\left\langle p_{\alpha}: \alpha<\delta\right\rangle$ is directed if for any two distinct elements $p_{\varrho}, p_{\nu} \in\left\langle p_{\alpha}: \alpha<\delta\right\rangle, p_{\varrho}$ and $p_{\nu}$ have a common upper bound of the form $p_{\sigma}$ ) there is an upper bound $p \in \mathbb{P}$. Furthermore, $\mathbb{P}$ is $\kappa$-strategically closed if in the two-person game in which the players construct an increasing sequence $\left\langle p_{\alpha}: \alpha \leq \kappa\right\rangle$, where player I plays odd stages and player II plays even and limit stages (choosing the trivial condition at stage 0 ), player II has a strategy which ensures the 
game can always be continued. Note that if $\mathbb{P}$ is $\kappa$-strategically closed and $f: \kappa \rightarrow V$ is a function in $V^{\mathbb{P}}$, then $f \in V$. Moreover, $\mathbb{P}$ is $<\kappa$-strategically closed if $\mathbb{P}$ is $\delta$-strategically closed for all cardinals $\delta<\kappa$; and $\mathbb{P}$ is $\prec \kappa$ strategically closed if in the two-person game in which the players construct an increasing sequence $\left\langle p_{\alpha}: \alpha<\kappa\right\rangle$, where player I plays odd stages and player II plays even and limit stages, player II has a strategy which ensures the game can always be continued.

Suppose that $\lambda$ is a limit ordinal of uncountable cofinality. A subset $S \subseteq$ $\lambda$ will be called a non-reflecting stationary set of ordinals if $S$ is stationary in $\lambda$, yet for no limit ordinal $\delta<\lambda$ of uncountable cofinality is $S \cap \delta$ stationary in $\delta$.

In the proofs of Theorems 1 and 2 we will use two different versions of a partial ordering originally due to Jensen for adding a non-reflecting stationary set of ordinals to a regular cardinal. The proofs of the basic properties of each of these partial orderings can essentially be found in [8], pages 435-437. For completeness and comprehensibility, we will include below in Lemmas 1.1-1.3 proofs of the basic properties for the partial ordering used in the proof of Theorem 2. Readers should then be able to transfer these proofs to the partial ordering used in the proof of Theorem 1.

Suppose now that $\kappa<\lambda$ are regular cardinals. The partial ordering $\mathbb{P}(\kappa, \lambda)$ used in the proof of Theorem 1 is the partial ordering for adding a non-reflecting stationary set of ordinals of cofinality $\kappa$ to $\lambda$. Specifically, $\mathbb{P}(\kappa, \lambda)=\{p:$ for some $\alpha<\lambda, p: \alpha \rightarrow\{0,1\}$ is a characteristic function of $S_{p}$, a subset of $\alpha$ not stationary at its supremum nor having any initial segment which is stationary at its supremum, so that $\beta \in S_{p}$ implies $\beta>\kappa$ and $\operatorname{cof}(\beta)=\kappa\}$, ordered by $q \geq p$ iff $q \supseteq p$ and $S_{p}=S_{q} \cap \sup \left(S_{p}\right)$, i.e., $S_{q}$ is an end extension of $S_{p}$. It is well known that for $G V$-generic over $\mathbb{P}(\kappa, \lambda)$ (see [8] or [13]), in $V[G]$, a non-reflecting stationary set $S=S[G]=\bigcup\left\{S_{p}\right.$ : $p \in G\} \subseteq \lambda$ of ordinals of cofinality $\kappa$ has been introduced, and the bounded subsets of $\lambda$ are the same as those in $V$. It is also virtually immediate that $\mathbb{P}(\kappa, \lambda)$ is $\kappa$-directed closed, and it can be shown (see [8] or [13]) that $\mathbb{P}(\kappa, \lambda)$ is $\prec \lambda$-strategically closed.

Suppose now that $\kappa$ is a Mahlo cardinal. The partial ordering $\mathbb{P}(\kappa)$ used in the proof of Theorem 2 is the partial ordering for adding a non-reflecting stationary set of ordinals of a certain type to $\kappa$. Specifically, $\mathbb{P}(\kappa)=\{p$ : for some $\alpha<\kappa, p: \alpha \rightarrow\{0,1\}$ is a characteristic function of $S_{p}$, a subset of $\alpha$ not stationary at its supremum nor having any initial segment which is stationary at its supremum, so that if $\beta<\sup \left(S_{p}\right)$ is inaccessible, then $S_{p}-S_{p}\lceil\beta$ is composed of ordinals of cofinality at least $\beta\}$, ordered by $q \geq p$ iff $q \supseteq p$ and $S_{p}=S_{q} \cap \sup \left(S_{p}\right)$, i.e., $S_{q}$ is an end extension of $S_{p}$.

Lemma 1.1. For any cardinal $\delta<\kappa, \mathbb{P}(\kappa)$ is $\delta$-strategically closed. 
Proof. Let $\delta<\kappa$ be given. Since $\kappa$ is a Mahlo cardinal, let $\gamma>\delta$, $\gamma<\kappa$ be inaccessible. Consider the two-person game in which at any even (successor) stage $\alpha+2$, player II's response to the condition $p_{\alpha+1}$ chosen by player I at stage $\alpha+1$ is to choose a condition $p_{\alpha+2}$ so that $\sup \left(S_{p_{\alpha+1}}\right) \notin$ $S_{p_{\alpha+2}}$ and so that $\sup \left(S_{p_{\alpha+2}}\right)>\max \left(\gamma, \sup \left(S_{p_{\alpha+1}}\right)\right)$. At limit stages $\lambda \leq \delta$, player II plays $\bigcup_{\alpha<\lambda} p_{\alpha}$. Readers can easily verify that this yields a winning strategy for II, since for any limit ordinal $\lambda \leq \delta<\gamma$ of uncountable cofinality, the limit points of $C_{\lambda}=\left\{\sup \left(S_{p_{\alpha}}\right): \alpha<\lambda\right\}$, having cofinality $<\delta$, form a club disjoint from $S_{p_{\lambda}}$.

Lemma 1.2. For any inaccessible cardinal $\delta<\kappa$, there is a partial ordering $\mathbb{P}(\kappa / \delta)$ dense in $\mathbb{P}(\kappa)$ so that $\mathbb{P}(\kappa / \delta)$ is $\delta$-directed closed.

Proof. If $\delta<\kappa$ is inaccessible, let $\mathbb{P}(\kappa / \delta)=\{p: p \in \mathbb{P}(\kappa)$ is either the characteristic function of the empty set or $p \in \mathbb{P}(\kappa)$ is so that $S_{p}$ contains an ordinal $>\delta\}$, ordered in the same way as $\mathbb{P}(\kappa)$ is. We claim that $\mathbb{P}(\kappa / \delta)$ is as desired.

Let $p \in \mathbb{P}$ be given. Choose $\gamma>\max \left(\sup \left(S_{p}\right), \delta\right), \gamma<\kappa$ an inaccessible cardinal. Let $q \geq p$ be so that $\gamma \in S_{q}$. Clearly, $q \in \mathbb{P}(\kappa / \delta)$, so $\mathbb{P}(\kappa / \delta)$ is dense in $\mathbb{P}(\kappa)$.

To see that $\mathbb{P}(\kappa / \delta)$ is $\delta$-directed closed, assume without loss of generality that $\lambda<\delta$ is an uncountable regular cardinal and $\left\{r_{\alpha}: \alpha<\lambda\right\}$ is a set of distinct mutually compatible elements of $\mathbb{P}(\kappa / \delta)$. It is clear that $r=$ $\bigcup_{\alpha<\lambda} r_{\alpha}$ is the characteristic function for a subset of some $\zeta<\kappa$. Further, for $\gamma<\sup \left(S_{r}\right)$, if $\gamma$ is inaccessible, then $S_{r}-S_{r}\lceil\gamma$ is composed of ordinals of cofinality at least $\gamma$. Thus, it suffices to show that there are sets $C_{r}$ and $C_{r}\left\lceil\beta\right.$ for $\beta<\lambda$ a limit ordinal of uncountable cofinality so that $C_{r}$ is club in $\sup \left(S_{r}\right), C_{r} \cap S_{r}=\emptyset, C_{r}\left\lceil\beta\right.$ is club in $\sup \left(S_{\cup_{\alpha<\beta} r_{\alpha}}\right)$, and $C_{r} \uparrow \beta \cap S_{\cup_{\alpha<\beta} r_{\alpha}}=\emptyset$. Such a set $C_{r}$ can be defined by looking at the sequence $\left\langle\sup \left(S_{r_{\alpha}}\right): \alpha<\lambda\right\rangle$, taking $C_{r}$ as the limit points of this sequence, and taking $C_{r}\lceil\beta$ for $\beta<\lambda$ a limit ordinal of uncountable cofinality as the limit points of $\left\langle\sup \left(S_{r_{\alpha}}\right)\right.$ : $\alpha<\beta\rangle$. This works since each element of $C_{r}$ must be an ordinal $>\delta$ having cofinality $<\lambda$, yet by the definition of $\mathbb{P}(\kappa)$, each element of $S_{r}-S_{r}\lceil\delta$ or of $S_{\bigcup_{\alpha<\beta} r_{\alpha}}-S_{\cup_{\alpha<\beta} r_{\alpha}}\lceil\delta$ must have cofinality at least $\delta$.

Lemma 1.3. Let $G$ be $V$-generic over $\mathbb{P}(\kappa)$. Then $S=S[G]=\bigcup\left\{S_{p}\right.$ : $p \in G\} \subseteq \kappa$ is stationary in $\kappa$.

Proof. Let $p \in \mathbb{P}(\kappa)$ be so that $p \Vdash$ " $\dot{C}$ is club in $\kappa$ ". We show that for some $q \geq p, q \Vdash$ "C $\cap \dot{S} \neq \emptyset$ ". To do this, we define a sequence $\left\langle q_{\alpha}: \alpha<\kappa\right\rangle$ satisfying the following properties:

1. $q_{0} \geq p$.

2. If $0 \leq \alpha<\beta<\kappa$, then $q_{\beta} \geq q_{\alpha}$.

3. For each $\alpha<\kappa$, there is an ordinal $\varrho_{\alpha}<\kappa$ so that $q_{\alpha} \Vdash$ " $\varrho_{\alpha} \in \dot{C}$ ". 
4. The sequence $\left\langle\varrho_{\alpha}: \alpha<\kappa\right\rangle$ is strictly increasing.

5. The sequences $\left\langle\sup \left(S_{q_{\alpha}}\right): \alpha<\kappa\right\rangle$ and $\left\langle\varrho_{\alpha}: \alpha<\kappa\right\rangle$ are "interweaved" in the sense that $\varrho_{0}<\sup \left(S_{q_{0}}\right)<\varrho_{1}<\sup \left(S_{q_{1}}\right)<\ldots<\varrho_{\beta+1}<$ $\sup \left(S_{q_{\beta+1}}\right)<\ldots<\varrho_{\lambda}=\sup \left(S_{q_{\lambda}}\right)<\varrho_{\lambda+1}<\sup \left(S_{q_{\lambda+1}}\right)<\ldots$, with equality between $\varrho_{\lambda}$ and $\sup \left(S_{q_{\lambda}}\right)$ at limit ordinals $\lambda$.

Using ideas from Lemma 1.1, it is easy to construct the sequences $\left\langle\sup \left(S_{q_{\alpha}}\right): \alpha<\kappa\right\rangle$ and $\left\langle\varrho_{\alpha}: \alpha<\kappa\right\rangle$. Whenever player I chooses a condition $p_{\alpha}$ so that $p_{\alpha} \Vdash$ " $\varrho_{\alpha} \in \dot{C}$ " and $\varrho_{\alpha}>\sup \left(\left\langle\varrho_{\beta}: \beta<\alpha\right\rangle\right)$, player II responds by choosing a condition $q_{\alpha} \geq p_{\alpha}$ so that $\sup \left(S_{q_{\alpha}}\right)>\max \left(\sup \left(S_{p_{\alpha}}\right), \varrho_{\alpha}\right)$ and $\sup \left(S_{p_{\alpha}}\right) \notin S_{q_{\alpha}}$. At limit stages $\lambda$, player II simply chooses $q_{\lambda}$ as $\bigcup_{\alpha<\lambda} q_{\alpha}$ and $\varrho_{\lambda}$ as $\sup \left(\left\langle\varrho_{\alpha}: \alpha<\lambda\right\rangle\right)$. Readers can verify for themselves that this construction yields sequences with the desired properties.

By construction, $C^{\prime}=\left\{\varrho_{\alpha}: \alpha<\kappa\right\}$ is a club in $\kappa$. Since $\kappa$ is a Mahlo cardinal, there is some ordinal $\lambda<\kappa$ so that $\varrho_{\lambda}$ is inaccessible and $\varrho_{\lambda} \in C^{\prime}$. We then deduce that $q=q_{\lambda} \cup\left\{\left\langle\varrho_{\lambda}, 1\right\rangle\right\}$ is so that $q \geq p$ and $q \Vdash " \dot{C} \cap \dot{S} \neq \emptyset "$.

We remark that the construction given in Lemma 1.3 for the sequences $\left\langle\sup \left(S_{q_{\alpha}}\right): \alpha<\kappa\right\rangle$ and $\left\langle\varrho_{\alpha}: \alpha<\kappa\right\rangle$ essentially shows that either $\mathbb{P}(\kappa)$ or $\mathbb{P}(\kappa / \delta)$ for $\delta<\kappa$ inaccessible is $\prec \kappa$-strategically closed. Also, we note that for the rest of the paper, if $\mathbb{P}$ is either a product, iteration, or some combination of the two which, for some cardinal $\kappa$, adds a non-reflecting stationary set of ordinals to $\kappa$, then we will say that $\kappa$ is in the support of $\mathbb{P}$.

2. The proof of Theorem 1. Let $V$ and $\mathfrak{K}$ be as in the hypotheses of Theorem 1. Let $\mathbb{P}^{0} \subseteq V$ be a (possibly proper class) partial ordering, defined as in [1], so that $V^{\mathbb{P}^{0}} \vDash$ "ZFC + Every $V$-supercompact cardinal $\kappa$ is supercompact + Every $V$-supercompact cardinal is Laver indestructible $[14]+$ The supercompact and strongly compact cardinals coincide precisely, except at measurable limit points". Since we may assume without loss of generality that $\mathbb{P}^{0}$ is defined as a reverse Easton iteration which begins by adding a Cohen subset to $\omega, \mathbb{P}^{0}$ can be written as $\mathbb{Q} * \dot{\mathbb{R}}$, where $|\mathbb{Q}|=\omega$ and $\vdash_{\mathbb{Q}}$ " $\mathbb{R}$ is $\aleph_{1}$-strategically closed". Thus, in Hamkins' terminology of [10]-[12], $\mathbb{P}^{0}$ "admits a gap at $\aleph_{1}$ ", so by the results of [10]-[12], $V^{\mathbb{P}^{0}} \vDash$ "The only supercompact cardinals are those that were supercompact in $V$ ", i.e., $V^{\mathbb{P}^{0}} \vDash$ "Every supercompact cardinal is Laver indestructible".

Work now in $V_{0}=V^{\mathbb{P}^{0}}$. Let $\mathfrak{D}=\left\langle\delta_{\alpha}: \alpha<\Omega\right\rangle$, where $\Omega$ is the appropriate ordinal if $\mathfrak{K}$ is a set but is the class of ordinals otherwise, enumerate in increasing order $\{\delta: \delta$ is either a measurable limit of supercompact cardinals or the cardinal successor of a non-measurable limit point of the measurable limits of supercompact cardinals $\} \cup\{\omega\}$. For each $\alpha<\Omega$, let $\mathbb{P}_{\alpha}$ be the Easton support iteration of the partial orderings which add, for each supercompact cardinal in the interval $\left(\delta_{\alpha}, \delta_{\alpha+1}\right)$, a non-reflecting stationary set 
of ordinals of cofinality $\delta_{\alpha}$ (which by definition is a regular cardinal). Let $\mathbb{P}^{1}$ be the Easton support product $\prod_{\alpha<\Omega} \mathbb{P}_{\alpha}$, and in $V$, let $\mathbb{P}=\mathbb{P}^{0} * \dot{\mathbb{P}}^{1}$. Standard arguments concerning class forcing combined with the definition of $\mathbb{P}^{1}$ yield that $V^{\mathbb{P}}=V_{0}^{\mathbb{P}^{1}} \vDash \mathrm{ZFC}$.

Lemma 2.1. $V^{\mathbb{P}}=V_{0}^{\mathbb{P}^{1}} \vDash$ "If $\kappa \in \mathfrak{K}$, then $\kappa$ is supercompact".

Proof. The proof of Lemma 2.1 uses ideas found in the proof of Lemma 9 of [4]. Fix $\kappa \in \mathfrak{K}$. Working in $V_{0}$, write $\mathbb{P}^{1}=\mathbb{Q}_{\kappa} \times \mathbb{Q}^{\kappa}$, where $\mathbb{Q}^{\kappa}=\prod_{\delta \geq \kappa} \mathbb{P}_{\delta}$, and $\mathbb{Q}_{\kappa}$ is the rest of $\mathbb{P}^{1}$. By the definition of $\mathbb{Q}^{\kappa}, V_{0} \vDash$ " $\mathbb{Q}^{\kappa}$ is $\kappa$-directed closed". Thus, by the construction of $V_{0}, V_{1}=V_{0}^{\mathbb{Q}^{\kappa}} \vDash " \kappa$ is supercompact". It therefore suffices to show that $V_{1}^{\mathbb{Q}_{\kappa}} \vDash$ " $\kappa$ is supercompact".

To do this, let $\lambda \geq \kappa$ be arbitrary, and let $\gamma=\left|2^{[\lambda]^{<\kappa}}\right|$. Let $j: V_{1} \rightarrow M$ be an elementary embedding witnessing the $\gamma$ supercompactness of $\kappa$ so that $M \vDash$ " $\kappa$ is not supercompact". We observe that any $\delta \in(\kappa, \gamma]$ must be so that $M \vDash$ " $\delta$ is not supercompact", for if this were not the case, then the fact $M^{\gamma} \subseteq M$ allows us to infer that $M \vDash " \kappa$ is $<\delta$-supercompact and $\delta$ is supercompact". Hence, a theorem of Magidor found in [17] tells us that $M \vDash " \kappa$ is supercompact", a contradiction. Writing $j\left(\mathbb{Q}_{\kappa}\right)=\mathbb{Q}_{\kappa} \times \mathbb{Q}^{*}$, the preceding says that in $M$, the least cardinal $\gamma_{0}$ in the support of $\mathbb{Q}^{*}$ must be so that $\gamma_{0}>\gamma$.

Let $G$ be $V_{1}$-generic over $\mathbb{Q}_{\kappa}$ and $H$ be $V_{1}[G]$-generic over $\mathbb{Q}^{*}$. In $V_{1}[G \times H], j: V_{1} \rightarrow M$ extends to $\bar{j}: V_{1}[G] \rightarrow M[G \times H]$ via the defi-

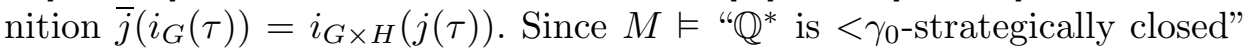
and $\gamma<\gamma_{0}$, the fact $M^{\gamma} \subseteq M$ implies $V_{1} \vDash " \mathbb{Q}^{*}$ is $\gamma$-strategically closed" yields that for any cardinal $\delta \leq \gamma, V_{1}[G]$ and $V_{1}[G \times H]=V_{1}[H \times G]$ contain the same subsets of $\delta$. This means the supercompact ultrafilter $\mathcal{U}$ over $\left(P_{\kappa}(\lambda)\right)^{V_{1}[G]}$ in $V_{1}[G \times H]$ given by $x \in \mathcal{U}$ iff $\langle j(\alpha): \alpha<\lambda\rangle \in \bar{j}(x)$ is so that $\mathcal{U} \in V_{1}[G]$.

LEMmA 2.2. $V^{\mathbb{P}}=V_{0}^{\mathbb{P}^{1}} \vDash$ "The only supercompact cardinals are the elements of $\mathfrak{K} "$.

Proof. In analogy to what was done in the first paragraph of the proof of Theorem 1 , write $\mathbb{P}=\mathbb{Q} * \dot{\mathbb{R}}$, where $|\mathbb{Q}|=\omega$ and $\vdash_{\mathbb{Q}}$ " $\mathbb{R}$ is $\aleph_{1}$-strategically closed". Thus, as before, $\mathbb{P}$ "admits a gap at $\aleph_{1}$ ", so by the results of [10][12], any supercompact cardinal in $V^{\mathbb{P}}$ had to have been supercompact in $V$. Hence, the proof of Lemma 2.2 will be complete once we have shown that any $V$-supercompact cardinal not in $\mathfrak{K}$ is no longer supercompact in $V^{\mathbb{P}}$.

Fix $\kappa$, a $V$-supercompact cardinal not an element of $\mathfrak{K}$. Work in $V_{0}$. By the definition of $\mathfrak{D}$, this means we can find $\alpha<\Omega$ so that $\kappa \in\left(\delta_{\alpha}, \delta_{\alpha+1}\right)$. Writing $\mathbb{P}_{\alpha}=\mathbb{Q}_{0} * \dot{\mathbb{Q}}_{1}$, where the least cardinal in the support of $\dot{\mathbb{Q}}_{1}$ is above $\kappa$, we know by the definition of $\mathbb{P}_{\alpha}$ that $V_{0}^{\mathbb{Q}_{0}} \vDash$ " $\kappa$ contains a non-reflecting stationary set of ordinals of cofinality $\delta_{\alpha}$ and therefore is not weakly compact 
and $\mathbb{Q}_{1}$ is $<\delta$-strategically closed for $\delta$ the least $V$-supercompact cardinal above $\kappa$ ". Hence, $V_{0}^{\mathbb{Q}_{0} * \dot{\mathbb{Q}}_{1}}=V_{0}^{\mathbb{P}_{\alpha}} \vDash$ " $\kappa$ contains a non-reflecting stationary set of ordinals of cofinality $\delta_{\alpha}$ and therefore is not weakly compact".

Write $\mathbb{P}^{1}=\mathbb{P}_{>\alpha} \times \mathbb{P}_{\alpha} \times \mathbb{P}_{<\alpha}$, where $\mathbb{P}_{>\alpha}$ is the Easton support product $\prod_{\beta \in(\alpha, \Omega)} \mathbb{P}_{\beta}$ and $\mathbb{P}_{<\alpha}$ is the Easton support product $\prod_{\beta<\alpha} \mathbb{P}_{\beta}$. By definition, in $V_{0}, \mathbb{P}_{>\alpha}$ is $\delta_{\alpha+1}$-strategically closed and $\left|\mathbb{P}_{<\alpha}\right|<\kappa$. Thus, $V_{0}^{\mathbb{P}>\alpha \times \mathbb{P}_{\alpha}} \vDash$ " $\kappa$ contains a non-reflecting stationary set of ordinals of cofinality $\delta_{\alpha}$ and therefore is not weakly compact", and by the Lévy-Solovay results [15], $V_{0}^{\mathbb{P}>\alpha} \times \mathbb{P}_{\alpha} \times \mathbb{P}_{<\alpha}=V_{0}^{\mathbb{P}^{1}}=V^{\mathbb{P}} \vDash " \kappa$ is not weakly compact (and hence is not supercompact)".

Lemma 2.3. $V^{\mathbb{P}}=V_{0}^{\mathbb{P}^{1}} \vDash$ "If $\kappa \in \mathfrak{K}$, then $\kappa$ is a limit of (non-supercompact) strongly compact cardinals".

Proof. Fix $\kappa \in \mathfrak{K}$. Since $\kappa$ is a supercompact limit of supercompact cardinals in $V_{0}$ (as well as in $V$ ), by Theorem 1 of [3], for any $\gamma<\kappa$, we can find a non-supercompact measurable limit of supercompact cardinals $\delta \in(\gamma, \kappa)$. For such a $\delta$, let $\alpha$ be so that $\delta=\delta_{\alpha}$. By Menas' theorem of [18], which essentially says that for any $\gamma$, the least measurable limit of either supercompact or strongly compact cardinals above $\gamma$ is a non-supercompact strongly compact cardinal, we know that $\delta_{\alpha+1}$ is not supercompact, i.e., $\delta_{\alpha+1} \in\left(\delta_{\alpha}, \kappa\right)$. The proof of Lemma 2.3 will thus be complete once we have shown that $V_{0}^{\mathbb{P}^{1}} \vDash " \delta_{\alpha+1}$ is strongly compact".

As in Lemma 2.2, in $V_{0}$, write $\mathbb{P}^{1}=\mathbb{P}_{>\alpha} \times \mathbb{P}_{\alpha} \times \mathbb{P}_{<\alpha}$. Since $V_{0} \vDash " \mathbb{P}_{>\alpha}$ is $\delta_{\alpha+1}$-directed closed and $\delta_{\alpha+1}^{+}$is a measurable limit of indestructible supercompact cardinals", $V_{0}^{\mathbb{P}>\alpha} \vDash " \delta_{\alpha+1}$ is a measurable limit of supercompact cardinals". And, by Lemma 2 of [4], for any cardinal $\gamma>\delta_{\alpha+1}$, there is a strongly compact ultrafilter $\mathcal{U}$ over $P_{\delta_{\alpha}+1}(\gamma)$ so that for $j_{\mathcal{U}}: V_{0}^{\mathbb{P}>\alpha} \rightarrow M$ the associated $\gamma$ strongly compact embedding and $g$ the function representing $\delta_{\alpha+1}$ in $M,\left\{p \in P_{\delta_{\alpha}+1}(\gamma): f^{\prime}(g(p))>|p|\right\} \in \mathcal{U}$, where $f^{\prime}: \delta_{\alpha+1} \rightarrow \delta_{\alpha+1}$, $f^{\prime} \in V_{0}^{\mathbb{P}>\alpha}$ is the function defined by $f^{\prime}(\beta)=$ the least strongly compact cardinal above $\beta$. Therefore, if $\gamma>\delta_{\alpha+1}$ is an arbitrary cardinal and $\lambda=\left|2^{[\gamma]^{<\delta_{\alpha+1}}}\right|$, we can use the arguments given in Lemma 4 of [4] to show that for $j_{\mathcal{U}}\left(\mathbb{P}_{\alpha}\right)=\mathbb{P}_{\alpha} * \dot{\mathbb{S}}$, if $G_{0}$ is $V_{0}^{\mathbb{P}>\alpha}$-generic over $\mathbb{P}_{\alpha}$ and $G_{1}$ is $V_{0}^{\mathbb{P}>\alpha}\left[G_{0}\right]$-generic over $\mathbb{S}$, then $j_{\mathcal{U}}: V_{0}^{\mathbb{P}>\alpha} \rightarrow M$ extends in $V_{0}^{\mathbb{P}>\alpha}\left[G_{0}\right]\left[G_{1}\right]$ to $j: V_{0}^{\mathbb{P}>\alpha}\left[G_{0}\right] \rightarrow M\left[G_{0}\right]\left[G_{1}\right]$. Consequently, there is a strongly compact ultrafilter $\mu$ over $\left(P_{\delta_{\alpha+1}}(\gamma)\right)^{V_{0}^{\mathbb{P}>\alpha}\left[G_{0}\right]}$ present in $V_{0}^{\mathbb{P}>\alpha}\left[G_{0}\right]\left[G_{1}\right]$, and by the choice of $\lambda$ and arguments found in Lemma 4 of $[4], \mu \in V_{0}^{\mathbb{P}>\alpha}\left[G_{0}\right]$. Since $\gamma$ was arbitrary, we thus know that $V_{0}^{\mathbb{P}_{>\alpha} \times \mathbb{P}_{\alpha}} \vDash " \delta_{\alpha+1}$ is strongly compact". Hence, since $V_{0} \vDash "\left|\mathbb{P}_{<\alpha}\right|<\delta_{\alpha+1}$ ", the results of [15] imply that $V_{0}^{\mathbb{P}>\alpha \times \mathbb{P}_{\alpha} \times \mathbb{P}_{<\alpha}}=V_{0}^{\mathbb{P}^{1}}=V^{\mathbb{P}} \vDash " \delta_{\alpha+1}$ is strongly compact". 
Lemmas 2.1-2.3 complete the proof of Theorem 1.

We conclude Section 2 by observing it is not true in the model $V^{\mathbb{P}}$ constructed above that every measurable cardinal is strongly compact. To see this, by the last paragraph of the proof of Lemma 2.2, if $\delta \in\left(\delta_{\alpha}, \delta_{\alpha+1}\right)$ is measurable, then $V_{0}^{\mathbb{P}_{>\alpha} \times \mathbb{P}_{\alpha}} \vDash$ "There is a $V$-supercompact cardinal $\kappa>\delta$ containing a non-reflecting stationary set of ordinals of cofinality $\delta_{\alpha}$ ". By Theorem 4.8 of [19] and the succeeding remarks, $V_{0}^{\mathbb{P}_{>\alpha} \times \mathbb{P}_{\alpha}} \vDash$ " $\delta$ is not strongly compact", so the results of [15] imply that $V_{0}^{\mathbb{P}>\alpha} \times \mathbb{P}_{\alpha} \times \mathbb{P}_{<\alpha}=V_{0}^{\mathbb{P}_{1}}=$ $V^{\mathbb{P}} \vDash$ " $\delta$ is not strongly compact". Indeed, the construction of a model from any hypotheses in which the first $\omega$ measurables are strongly compact is the main question left open in [13]. However, in Section 3, the model constructed will be so that every measurable cardinal has some non-trivial degree of strong compactness.

3. The proof of Theorem 2. Let $V$ and $\mathfrak{K}$ be as in the hypotheses for Theorem 2. Without loss of generality, by first doing a preliminary forcing if necessary, we may assume that $V \vDash \mathrm{GCH}$ as well.

Let $\mathfrak{D}=\left\langle\delta_{\alpha}: \alpha<\Omega\right\rangle$ enumerate in increasing order $\{\delta: \delta$ is a measurable cardinal which is not $2^{\delta}$ supercompact $\}$, where $\Omega$ is the appropriate ordinal if the collection of all measurable cardinals forms a set or is the class of all ordinals otherwise. The partial ordering $\mathbb{P}$ used in the proof of Theorem 2 is the Easton support iteration which first adds a Cohen subset to $\omega$ and then adds, to every $\delta \in \mathfrak{D}$, a non-reflecting stationary set of ordinals using the partial ordering $\mathbb{P}(\delta)$ described in Section 1. Note that no $\kappa \in \mathfrak{K}$ will be an element of the support of $\mathbb{P}$. Also, as before, standard arguments concerning class forcing combined with the definition of $\mathbb{P}$ yield that $V^{\mathbb{P}} \vDash \mathrm{ZFC}$.

LEMma 3.1. $V^{\mathbb{P}} \vDash$ "The only supercompact cardinals are the elements of $\mathfrak{K} "$.

Proof. As in the first paragraphs of the proofs of Theorem 1 and Lemma 2.2 , write $\mathbb{P}=\mathbb{P}^{\prime} * \dot{\mathbb{P}}^{\prime \prime}$, where $\left|\mathbb{P}^{\prime}\right|=\omega$ and $\Vdash_{\mathbb{P}^{\prime}}$ " $\dot{\mathbb{P}}^{\prime \prime}$ is $\aleph_{1}$-strategically closed". Hence, again as earlier, $\mathbb{P}$ "admits a gap at $\aleph_{1}$ ", so by the results of [10]-[12], any supercompact cardinal in $V^{\mathbb{P}}$ had to have been supercompact in $V$. This means the proof of Lemma 3.1 will be complete once we have shown that $V^{\mathbb{P}} \vDash$ "If $\kappa \in \mathfrak{K}$, then $\kappa$ is supercompact".

To do this, fix $\kappa \in \mathfrak{K}$, and let $\lambda>\kappa$ be an arbitrary successor cardinal. Let $\gamma=\left|2^{[\lambda]^{<\kappa}}\right|$, and fix an elementary embedding $j: V \rightarrow M$ witnessing the $\gamma$ supercompactness of $\kappa$.

Write $\mathbb{P}=\mathbb{P}^{0} * \dot{\mathbb{P}}^{1} * \dot{\mathbb{P}}^{2}$, where $\mathbb{P}^{0}$ is the portion of $\mathbb{P}$ defined through stage $\kappa, \dot{\mathbb{P}}^{1}$ is a term for the portion of $\mathbb{P}$ defined between stages $\kappa$ and $\lambda$, and $\dot{\mathbb{P}}^{2}$ is a term for the rest of $\mathbb{P}$. By the definition of $\mathbb{P}$, it will be the case 
that $\Vdash_{\mathbb{P}^{0} * \dot{\mathbb{P}}^{1}}$ " $\dot{\mathbb{P}}^{2}$ is $\gamma$-strategically closed". Thus, it will suffice to show that $V^{\mathbb{P}^{0} * \dot{\mathbb{P}}^{1}} \vDash$ " $\kappa$ is $\lambda$ supercompact".

If this is not the case, then let $p=\left\langle p_{0}, \dot{p}_{1}\right\rangle \in \mathbb{P}^{0} * \dot{\mathbb{P}}^{1}$ be so that $p \Vdash$ " $\kappa$ is not $\lambda$ supercompact". By using Lemma 1.2 if necessary to find the necessary terms to extend coordinatewise, we assume without loss of generality that each non-trivial coordinate of $p_{1}$ is a term for a condition in the appropriate $\mathbb{P}(\delta / \kappa)$.

Let $G_{0}$ be $V$-generic over $\mathbb{P}^{0}$ so that $p_{0} \in G_{0}$. Working in $V\left[G_{0}\right]$ and once again using Lemma 1.2 , let $\mathbb{P}^{3}$ be the Easton support iteration of partial orderings which, for every measurable $\delta \in(\kappa, \lambda)$ which is not $2^{\delta}$ supercompact (in either $V$ or $V\left[G_{0}\right]$ ), add non-reflecting stationary sets of ordinals using $\mathbb{P}(\delta / \kappa)$.

Note now that if $G_{1}$ is $V\left[G_{0}\right]$-generic over $\mathbb{P}^{3}$ and $p_{1} \in G_{1}$, then $G_{1}$ must also generate a $V\left[G_{0}\right]$-generic filter $G_{1}^{*}$ over $\mathbb{P}^{1}$. To see this, it clearly suffices to show that $G_{1}$ meets all dense open subsets of $\mathbb{P}^{1}$ above $p_{1}$. If $D$ is such a set, then let $D_{1}=\left\{q \in \mathbb{P}^{3}: q\right.$ extends some element of $\left.D\right\}$. $D_{1}$ is clearly open. If $q \in \mathbb{P}^{3}$, then $q \in \mathbb{P}^{1}$, so by density, there is $q^{\prime} \geq q$, $q^{\prime} \in D$. By using Lemma 1.2 if necessary to find a term which is forced to extend each term denoting a non-trivial coordinate of $q^{\prime}$ to a term for an element of the appropriate $\mathbb{P}(\delta / \kappa)$, we obtain $q^{\prime \prime} \geq q^{\prime} \geq q, q^{\prime \prime} \in D_{1}$. Thus, $G_{1}$ meets $D_{1}$ and hence meets $D$, so $G_{1}$ generates a $V\left[G_{0}\right]$-generic filter $G_{1}^{*}$ over $\mathbb{P}^{1}$.

By the definition of $\mathbb{P}$ and the closure properties of $M, j\left(\mathbb{P}^{0} * \dot{\mathbb{P}}^{1}\right)=\mathbb{P}^{0} *$ $\dot{\mathbb{P}}^{1} * \dot{\mathbb{Q}} * \dot{\mathbb{R}}$, where $\dot{\mathbb{Q}}$ is a term for the portion of $j\left(\mathbb{P}^{0} * \dot{\mathbb{P}}^{1}\right)$ defined in $M$ between stages $\lambda$ and $j(\kappa)$, and $\dot{\mathbb{R}}$ is a term for $j\left(\dot{\mathbb{P}}^{1}\right)$, i.e., the portion of $j\left(\mathbb{P}^{0} * \dot{\mathbb{P}}^{1}\right)$ defined in $M$ between stages $j(\kappa)$ and $j(\lambda)$. If $G_{1}$ is $V\left[G_{0}\right]$-generic over $\mathbb{P}^{3}$ and $p_{1} \in G_{1}$, then by the preceding paragraph, $G_{1}$ generates a $V\left[G_{0}\right]$-generic filter $G_{1}^{*}$ over $\mathbb{P}^{1}$. We can therefore take $G_{2}$ as a $V\left[G_{0}\right]\left[G_{1}^{*}\right]$-generic object over $\mathbb{Q}$ and use the usual Easton arguments to infer that $M\left[G_{0}\right]\left[G_{1}^{*}\right]\left[G_{2}\right]$ remains $\gamma$ closed with respect to $V\left[G_{0}\right]\left[G_{1}^{*}\right]\left[G_{2}\right]$ and that $j$ extends in $V\left[G_{0}\right]\left[G_{1}^{*}\right]\left[G_{2}\right]$ to $j: V\left[G_{0}\right] \rightarrow M\left[G_{0}\right]\left[G_{1}^{*}\right]\left[G_{2}\right]$. Further, since $G_{1} \subseteq G_{1}^{*}$ and $G_{1}$ is $V\left[G_{0}\right]$-generic over a partial ordering $\left(\mathbb{P}^{3}\right)$ that is $\kappa$-directed closed in $V\left[G_{0}\right], j^{\prime \prime} G_{1}$ generates in $V\left[G_{0}\right]\left[G_{1}^{*}\right]\left[G_{2}\right]$ a compatible set of conditions of cardinality smaller than $\gamma<j(\kappa)$ in a partial ordering $\left(j\left(\mathbb{P}^{3}\right)\right)$ that is $j(\kappa)$-directed closed in $M\left[G_{0}\right]\left[G_{1}^{*}\right]\left[G_{2}\right]$. Therefore, since $M\left[G_{0}\right]\left[G_{1}^{*}\right]\left[G_{2}\right]$ is $\gamma$ closed with respect to $V\left[G_{0}\right]\left[G_{1}^{*}\right]\left[G_{2}\right]$, we can let $r$ be a master condition for $j^{\prime \prime} G_{1}$ and take $G_{3}$ to be a $V\left[G_{0}\right]\left[G_{1}^{*}\right]\left[G_{2}\right]$-generic object over $j\left(\mathbb{P}^{3}\right)$ containing $r$. By elementarity, it will be the case that $G_{3}$ generates a $V\left[G_{0}\right]\left[G_{1}^{*}\right]\left[G_{2}\right]$-generic object $G_{3}^{*}$ over $\mathbb{R}=j\left(\mathbb{P}^{1}\right)$. As usual, we will then have that in $V\left[G_{0}\right]\left[G_{1}^{*}\right]\left[G_{2}\right]\left[G_{3}^{*}\right], j$ extends to $j: V\left[G_{0}\right]\left[G_{1}^{*}\right] \rightarrow M\left[G_{0}\right]\left[G_{1}^{*}\right]\left[G_{2}\right]\left[G_{3}^{*}\right]$, so $\kappa$ is $\lambda$ supercompact in $V\left[G_{0}\right]\left[G_{1}^{*}\right]\left[G_{2}\right]\left[G_{3}^{*}\right]$. Since $\mathbb{Q} * \dot{\mathbb{R}}$ is $\gamma$-directed closed in $V\left[G_{0}\right]\left[G_{1}^{*}\right]$, it will be 
the case that $\kappa$ is $\lambda$ supercompact in $V\left[G_{0}\right]\left[G_{1}^{*}\right]$. This, however, contradicts the facts that $p=\left\langle p_{0}, p_{1}\right\rangle \in G_{0} * G_{1}^{*}$ and $p \Vdash$ " $\kappa$ is not $\lambda$ supercompact".

Lemma 3.2. $V^{\mathbb{P}} \vDash$ "For every cardinal $\delta, \delta$ is measurable iff $\delta$ is $2^{\delta}$ strongly compact".

Proof. Suppose $V^{\mathbb{P}} \vDash$ " $\delta$ is measurable". By Hamkins' work of [10][12], it must be true that $V \vDash " \delta$ is measurable". If $V \vDash$ " $\delta$ is not $2^{\delta}$ supercompact", write $\mathbb{P}=\mathbb{P}^{0} * \dot{\mathbb{P}^{1}}$, where $V^{\mathbb{P}^{0}} \vDash$ " $\delta$ contains a non-reflecting stationary set of ordinals and hence is not weakly compact". Since $\vdash_{\mathbb{P} 0}$ " $\mathbb{P}^{1}$ is $<\gamma$-strategically closed for $\gamma$ the least $V$ - or $V^{\mathbb{P}^{0}}$-measurable cardinal above $\delta ", V^{\mathbb{P}^{0} * \dot{\mathbb{P}}^{1}}=V^{\mathbb{P}} \vDash " \delta$ contains a non-reflecting stationary set of ordinals and hence is not measurable or weakly compact".

We therefore know that $V \vDash " \delta$ is $2^{\delta}$ supercompact". We can then write $\mathbb{P}=\mathbb{P}^{0} * \dot{\mathbb{P}}^{1}$, where the support of $\mathbb{P}^{0}$ consists of all measurable cardinals $\gamma<\delta$ which are not $2^{\gamma}$ supercompact. Since as in the preceding paragraph, $\Vdash_{\mathbb{P}^{0}}$ " $\dot{\mathbb{P}}^{1}$ is $<\gamma$-strategically closed for $\gamma$ the least $V$ - or $V^{\mathbb{P}^{0}}$-measurable cardinal above $\delta ", V^{\mathbb{P}^{0} * \dot{\mathbb{P}}^{1}}=V^{\mathbb{P}} \vDash " \delta$ is $2^{\delta}$ strongly compact" iff $V^{\mathbb{P}^{0}} \vDash " \delta$ is $2^{\delta}$ strongly compact".

To show $V^{\mathbb{P}^{0}} \vDash$ " $\delta$ is $2^{\delta}$ strongly compact", we use an unpublished argument of Magidor. Although the essentials of this argument can be found in [5] and [6], for completeness and for the benefit of the readers, we give the argument here as well. Let $\lambda=2^{\delta}=\delta^{+}$, and let $k_{1}: V \rightarrow M$ be an embedding witnessing the $\lambda$ supercompactness of $\delta$ so that $M \vDash " \delta$ is not $\lambda$ supercompact". Since $M \vDash$ " $\delta$ is measurable", we may choose a normal ultrafilter of Mitchell order 0 over $\delta$ so that $k_{2}: M \rightarrow N$ is an embedding witnessing the measurability of $\delta$ definable in $M$ with $N \vDash$ " $\delta$ is not measurable". It is the case that if $k: V \rightarrow N$ is an elementary embedding with critical point $\delta$ and for any $x \subseteq N$ with $|x| \leq \lambda$, there is some $y \in N$ so that $x \subseteq y$ and $N \vDash "|y|<k(\delta)$ ", then $k$ witnesses the $\lambda$ strong compactness of $\delta$. Using this fact, it is easily verifiable that $j=k_{2} \circ k_{1}$ is an elementary embedding witnessing the $\lambda$ strong compactness of $\delta$. We show that $j$ extends to $j: V^{\mathbb{P}^{0}} \rightarrow N^{j\left(\mathbb{P}^{0}\right)}$. Since this extended embedding witnesses the $\lambda$ strong compactness of $\delta$ in $V^{\mathbb{P}^{0}}$, this proves Lemma 3.2.

To do this, write $j\left(\mathbb{P}^{0}\right)$ as $\mathbb{P}^{0} * \dot{\mathbb{Q}}^{0} * \dot{\mathbb{R}}^{0}$, where $\dot{\mathbb{Q}}^{0}$ is a term for the portion of $j\left(\mathbb{P}^{0}\right)$ between $\delta$ and $k_{2}(\delta)$ and $\dot{\mathbb{R}}^{0}$ is a term for the rest of $j\left(\mathbb{P}^{0}\right)$, i.e., the part above $k_{2}(\delta)$. Note that since $N \vDash$ " $\delta$ is not measurable", $\delta \notin \operatorname{support}\left(\dot{\mathbb{Q}}^{0}\right)$. Thus, the support of $\dot{\mathbb{Q}}^{0}$ is composed of all $N$-measurable cardinals $\gamma$ in the interval $\left(\delta, k_{2}(\delta)\right]$ which are not $2^{\gamma}$ supercompact (so $k_{2}(\delta) \in \operatorname{support}\left(\dot{\mathbb{Q}}^{0}\right)$ since $M \vDash " \delta$ is measurable but $\delta$ is not $2^{\delta}$ supercompact"), and the support of $\dot{\mathbb{R}}^{0}$ is composed of all $N$-measurable cardinals $\gamma$ in the interval $\left(k_{2}(\delta), k_{2}\left(k_{1}(\delta)\right)\right)$ which are not $2^{\gamma}$ supercompact. 
Let $G_{0}$ be $V$-generic over $\mathbb{P}^{0}$. We construct in $V\left[G_{0}\right]$ an $N\left[G_{0}\right]$-generic object $G_{1}$ over $\mathbb{Q}^{0}$ and an $N\left[G_{0}\right]\left[G_{1}\right]$-generic object $G_{2}$ over $\mathbb{R}^{0}$. Since $\mathbb{P}^{0}$ is an Easton support iteration of length $\delta$, a direct limit is taken at stage $\delta$, and no forcing is done at stage $\delta$, the construction of $G_{1}$ and $G_{2}$ automatically guarantees that $j^{\prime \prime} G_{0} \subseteq G_{0} * G_{1} * G_{2}$. This means that $j: V \rightarrow N$ extends to $j: V\left[G_{0}\right] \rightarrow N\left[G_{0}\right]\left[G_{1}\right]\left[G_{2}\right]$.

To build $G_{1}$, note that since $k_{2}$ can be assumed to be generated by an ultrafilter $\mathcal{U}$ over $\delta$ and since $2^{\delta}=\delta^{+}$in both $V$ and $M$, we have $\left|k_{2}\left(\delta^{+}\right)\right|=\left|k_{2}\left(2^{\delta}\right)\right|=\mid\left\{f: f: \delta \rightarrow \delta^{+}\right.$is a function $\}|=|\left[\delta^{+}\right]^{\delta} \mid=\delta^{+}$. Thus, as $N\left[G_{0}\right] \vDash "\left|\wp\left(\mathbb{Q}^{0}\right)\right|=k_{2}\left(2^{\delta}\right)$ ", we can let $\left\langle D_{\alpha}: \alpha<\delta^{+}\right\rangle$enumerate in $V\left[G_{0}\right]$ the dense open subsets of $\mathbb{Q}^{0}$ found in $N\left[G_{0}\right]$. For the purpose of the construction of $G_{1}$ to be given below, we further assume that for every dense open subset $D \subseteq \mathbb{Q}^{0}$ present in $N\left[G_{0}\right]$, for some odd ordinal $\gamma+1$, $D=D_{\gamma+1}$. Since the $\delta$ closure of $N$ with respect to either $M$ or $V$ implies the least element of the support of $\mathbb{Q}^{0}$ is $>\delta^{+}$, the definition of $\mathbb{Q}^{0}$ as the Easton support iteration which adds a non-reflecting stationary set of ordinals to each $N\left[G_{0}\right]$-measurable cardinal $\gamma$ in the interval $\left(\delta, k_{2}(\delta)\right]$ which is not $2^{\gamma}$ supercompact implies that $N\left[G_{0}\right] \vDash " \mathbb{Q}^{0}$ is $\prec \delta^{+}$-strategically closed". The standard arguments show that forcing with the $\delta$-c.c. partial ordering $\mathbb{P}^{0}$ preserves that $N\left[G_{0}\right]$ remains $\delta$-closed with respect to either $M\left[G_{0}\right]$ or $V\left[G_{0}\right]$; consequently, $\mathbb{Q}^{0}$ is $\prec \delta^{+}$-strategically closed in both $M\left[G_{0}\right]$ and $V\left[G_{0}\right]$.

We can now construct $G_{1}$ in either $M\left[G_{0}\right]$ or $V\left[G_{0}\right]$ as follows. Players I and II play a game of length $\delta^{+}$. The initial pair of moves is generated by player II choosing the trivial condition $q_{0}$ and player I responding by choosing $q_{1} \in D_{1}$. Then, at an even stage $\alpha+2$, player II picks $q_{\alpha+2} \geq q_{\alpha+1}$ by using some fixed strategy $\mathcal{S}$, where $q_{\alpha+1}$ was chosen by player I to be so that $q_{\alpha+1} \in D_{\alpha+1}$ and $q_{\alpha+1} \geq q_{\alpha}$. If $\alpha$ is a limit ordinal, player II uses $\mathcal{S}$ to pick $q_{\alpha}$ extending each $q_{\beta}$ for $\beta<\alpha$. By the $\prec \delta^{+}$-strategic closure of $\mathbb{Q}^{0}$ in both $M\left[G_{0}\right]$ and $V\left[G_{0}\right]$, the sequence $\left\langle q_{\alpha}: \alpha<\delta^{+}\right\rangle$as just described exists. By construction, $G_{1}=\left\{p \in \mathbb{Q}^{0}: \exists \alpha<\delta^{+}\left[q_{\alpha} \geq p\right]\right\}$ is our $N\left[G_{0}\right]$-generic object over $\mathbb{Q}^{0}$.

It remains to construct in $V\left[G_{0}\right]$ the desired $N\left[G_{0}\right]\left[G_{1}\right]$-generic object $G_{2}$ over $\mathbb{R}^{0}$. To do this, we first note that as $M \vDash$ " $\delta$ is measurable but $\delta$ is not $2^{\delta}$ supercompact", we can write $k_{1}\left(\mathbb{P}^{0}\right)$ as $\mathbb{P}^{0} * \dot{\mathbb{S}}^{0} * \dot{\mathbb{T}}^{0}$, where $\Vdash_{\mathbb{P}^{0}}$ " $\dot{\mathbb{S}}^{0}=\dot{\mathbb{P}}(\delta)$ ", and $\dot{\mathbb{T}}^{0}$ is a term for the rest of $k_{1}\left(\mathbb{P}^{0}\right)$.

Note now that $M \vDash$ "No cardinal $\gamma \in(\delta, \lambda]$ is measurable". Thus, the support of $\dot{\mathbb{T}}^{0}$ is composed of all $M$-measurable cardinals $\gamma$ in the interval $\left(\lambda, k_{1}(\delta)\right)$ which are not $2^{\gamma}$ supercompact, which implies that in $M, \Vdash_{\mathbb{P}^{0} * \dot{\mathbb{S}}^{0}}$ " $\mathbb{T}^{0}$ is $\prec \lambda^{+}$-strategically closed". Further, since $V \vDash$ GCH and $\lambda$ is regular, $\left|[\lambda]^{<\delta}\right|=\lambda$ and $2^{\lambda}=\lambda^{+}$. Therefore, as $k_{1}$ can be assumed to be generated 
by an ultrafilter $\mathcal{U}$ over $P_{\delta}(\lambda),\left|k_{1}(\lambda)\right|=\left|k_{1}\left(\delta^{+}\right)\right|=\left|k_{1}\left(2^{\delta}\right)\right|=\left|2^{k_{1}(\delta)}\right|=$ $\mid\left\{f: f: P_{\delta}(\lambda) \rightarrow \delta^{+}\right.$is a function $\}|=|\left[\delta^{+}\right]^{\lambda}|=|[\lambda]^{\lambda} \mid=\lambda^{+}$.

Work until otherwise specified in $M$. Consider the "term forcing" partial ordering $\mathbb{T}^{*}$ (see [9], Section 1.2.5, page 8) associated with $\dot{\mathbb{T}}^{0}$, i.e., $\tau \in \mathbb{T}^{*}$ iff $\tau$ is a term in the forcing language with respect to $\mathbb{P}^{0} * \dot{\mathbb{S}}^{0}$ and $\vdash_{\mathbb{P}^{0} * \dot{S}^{0}}$ " $\tau \in \dot{\mathbb{T}}^{0}$ ", ordered by $\tau \geq \sigma$ iff $\Vdash_{\mathbb{P}^{0} * \dot{\mathbb{S}}^{0}}$ " $\tau \geq \sigma$ ". Although $\mathbb{T}^{*}$ as defined is technically a proper class, it is possible to restrict the terms appearing in it to a sufficiently large set-sized collection, with the additional crucial property that any term $\tau$ forced to be in $\dot{\mathbb{T}}^{0}$ is also forced to be equal to an element of $\mathbb{T}^{*}$. As we will show below, this can be done in such a way that $M \vDash "\left|\mathbb{T}^{*}\right|=k_{1}(\delta) "$.

Clearly, $\mathbb{T}^{*} \in M$. Also, since $\Vdash_{\mathbb{P}^{0} * \dot{\mathbb{S}}^{0}}$ " $\dot{\mathbb{T}}^{0}$ is $\prec \lambda^{+}$-strategically closed", it can easily be verified that $\mathbb{T}^{*}$ itself is $\prec \lambda^{+}$-strategically closed in $M$ and, since $M^{\lambda} \subseteq M$, in $V$ as well.

Observe that $M \vDash " k_{1}(\delta)$ is measurable and $\left|\mathbb{P}^{0} * \dot{\mathbb{S}}^{0}\right|<k_{1}(\delta)$ " and $\Vdash_{\mathbb{P}^{0} * \dot{\mathbb{S}}^{0}}$ " $\mathbb{T}^{0}$ is an Easton support iteration of length $k_{1}(\delta)$ and $\left|\dot{\mathbb{T}}^{0}\right|=k_{1}(\delta)$ ". We can thus let $\dot{f}$ be a term so that $\Vdash_{\mathbb{P}^{0} * \dot{\mathbb{S}}^{0}}$ " $\dot{f}: k_{1}(\delta) \rightarrow \dot{\mathbb{T}}^{0}$ is a bijection". Since $M \vDash "\left|\mathbb{P}^{0} * \dot{\mathbb{S}}^{0}\right|<k_{1}(\delta)$ ", for each $\alpha<k_{1}(\delta)$ let $S_{\alpha}=\left\{r_{\beta}^{\alpha}: \beta<\eta^{\alpha}<k_{1}(\delta)\right\}$ be a maximal incompatible set of elements of $\mathbb{P}^{0} * \dot{\mathbb{S}}^{0}$ so that for some term $\tau_{\beta}^{\alpha}, r_{\beta}^{\alpha} \Vdash$ " $\tau_{\beta}^{\alpha}=\dot{f}(\alpha)$ ". Define $T_{\alpha}=\left\{\tau_{\beta}^{\alpha}: \beta<\eta^{\alpha}\right\}$ and $T=\bigcup_{\alpha<k_{1}(\delta)} T_{\alpha}$. Clearly, $|T|=k_{1}(\delta)$, so we can let $\left\langle\tau_{\alpha}: \alpha<k_{1}(\delta)\right\rangle$ enumerate the members of $T$. Now $\left\langle\tau_{\alpha}: \alpha<k_{1}(\delta)\right\rangle$ has the property that if $\Vdash_{\mathbb{P}^{0} * \dot{\mathbb{S}}^{0}}$ " $\tau \in \dot{\mathbb{T}}^{0}$ ", then for some $\alpha<k_{1}(\delta), \Vdash_{\mathbb{P}^{0} * \dot{\mathbb{S}}^{0}}$ " $\tau=\tau_{\alpha}$ ". Therefore, we can restrict the set of terms we choose so that we can assume that in $M,\left|\mathbb{T}^{*}\right|=k_{1}(\delta)$. Since $M \vDash " 2^{k_{1}(\delta)}=\left(k_{1}(\delta)\right)^{+}=k_{1}\left(\delta^{+}\right)=k_{1}(\lambda)$ ", this means we can let $\left\langle D_{\alpha}: \alpha<\lambda^{+}\right\rangle$enumerate in $V$ the dense open subsets of $\mathbb{T}^{*}$ found in $M$, so that as before, every dense open subset $D \subseteq \mathbb{T}^{*}$ present in $M$ is $D_{\gamma+1}$ for some odd ordinal $\gamma+1$, and argue as we did earlier to construct in $V$ an $M$-generic object $\mathrm{H}_{2}$ over $\mathbb{T}^{*}$.

Note now that since $N$ can be assumed to be given by an ultrapower of $M$ via a normal ultrafilter $\mathcal{U} \in M$ over $\delta$, Fact 2 of Section 1.2.2 of [9] tells us that $k_{2}^{\prime \prime} H_{2}$ generates an $N$-generic object $G_{2}^{*}$ over $k_{2}\left(\mathbb{T}^{*}\right)$. By elementariness, $k_{2}\left(\mathbb{T}^{*}\right)$ is the term forcing in $N$ defined with respect to $k_{2}\left(k_{1}\left(\mathbb{P}_{\delta}\right)_{\delta+1}\right)=\mathbb{P}^{0} * \dot{\mathbb{Q}}^{0}$. Therefore, since $j\left(\mathbb{P}^{0}\right)=k_{2}\left(k_{1}\left(\mathbb{P}^{0}\right)\right)=\mathbb{P}^{0} * \dot{\mathbb{Q}}^{0} * \dot{\mathbb{R}}^{0}$, and $G_{2}^{*}$ is $N$-generic over $k_{2}\left(\mathbb{T}^{*}\right)$, and $G_{0} * G_{1}$ is $k_{2}\left(\mathbb{P}^{0} * \dot{\mathbb{S}}^{0}\right)$-generic over $N$, Fact 1 of Section 1.2.5 of [9] tells us that for $G_{2}=\left\{i_{G_{0} * G_{1}}(\tau): \tau \in G_{2}^{*}\right\}$, $G_{2}$ is $N\left[G_{0}\right]\left[G_{1}\right]$-generic over $\mathbb{R}^{0}$. Thus, in $V\left[G_{0}\right], j: V \rightarrow N$ extends to $j: V\left[G_{0}\right] \rightarrow N\left[G_{0}\right]\left[G_{1}\right]\left[G_{2}\right]$. As $V^{\mathbb{P}^{0}} \vDash$ " $\delta$ is $2^{\delta}$ strongly compact", this completes the proof of Lemma 3.2.

Lemmas 3.1 and 3.2 complete the proof of Theorem 2. 
We conclude Section 3 and this paper with two remarks. First, we observe we can guarantee that in Theorem 2, there are measurable cardinals $\delta$ which are not strongly compact. If we force over models such as those given in [1], [7], or [13] in which the classes of strongly compact and supercompact cardinals coincide precisely except at measurable limit points, then there will be many measurable cardinals, such as those below the least supercompact cardinal, which will not be strongly compact. If we force using the partial ordering $\mathbb{P}$ as just described, then by the work of [10]-[12], no new strongly compact cardinals are created. Thus, any cardinal which was not strongly compact in $V$ is not strongly compact in $V^{\mathbb{P}}$.

Second, we note that in Theorem 2, it is possible to have each measurable cardinal $\delta$ exhibit a greater degree of strong compactness than just $2^{\delta}$ strong compactness. The proof given in Lemma 3.2 will work for any regular value of $\lambda$ below the least $V$-measurable cardinal above $\delta$. Further, the nature of the partial ordering $\mathbb{P}$ used in the proof of Theorem 2 ensures that the cardinal structure between any $V$-measurable cardinal $\delta$ and the least $V$-measurable cardinal $\delta^{\prime}$ above it remains the same in $V^{\mathbb{P}}$, regardless of whether the measurability of either $\delta$ or $\delta^{\prime}$ is destroyed by $\mathbb{P}$. Thus, e.g., it is possible to construct a model in which the ground model and the generic extension have the same class $\mathfrak{K}$ of supercompact cardinals and $\delta$ is measurable iff $\delta$ is $\lambda$ strongly compact for $\lambda$ assuming values such as $2^{2^{\delta}}, \delta^{+17}$, the least inaccessible cardinal above $\delta$, the least weakly compact cardinal above $\delta$, etc. In certain of these models, it may be necessary to assume additional large cardinal hypotheses, e.g., if there is only one supercompact cardinal $\kappa$ in the universe and we wish every measurable cardinal $\delta$ to be $\lambda$ strongly compact for $\lambda$ the least inaccessible cardinal above $\delta$, it will be necessary to assume the existence of an inaccessible cardinal above $\kappa$.

\section{References}

[1] A. Apter, Laver indestructibility and the class of compact cardinals, J. Symbolic Logic 63 (1998), 149-157.

[2] -, Measurability and degrees of strong compactness, ibid. 46 (1981), 180-185.

[3] - On the least strongly compact cardinal, Israel J. Math. 35 (1980), 225-233.

[4] - Patterns of compact cardinals, Ann. Pure Appl. Logic 89 (1997), 101-115.

[5] A. Apter and J. Cummings, Identity crises and strong compactness, J. Symbolic Logic, to appear.

[6] -, 一, Identity crises and strong compactness II: strong cardinals, Arch. Math. Logic, to appear.

[7] A. Apter and S. Shelah, On the strong equality between supercompactness and strong compactness, Trans. Amer. Math. Soc. 349 (1997), 103-128.

[8] J. Burgess, Forcing, in: Handbook of Mathematical Logic, J. Barwise (ed.), NorthHolland, Amsterdam, 1977, 403-452. 
[9] J. Cummings, A model in which GCH holds at successors but fails at limits, Trans. Amer. Math. Soc. 329 (1992), 1-39.

[10] J. D. Hamkins, Destruction or preservation as you like it, Ann. Pure Appl. Logic 91 (1998), 191-229.

[11] —, Gap forcing, Israel J. Math., to appear.

[12] —, Gap forcing: generalizing the Lévy-Solovay theorem, Bull. Symbolic Logic 5 (1999), 264-272.

[13] Y. Kimchi and M. Magidor, The independence between the concepts of compactness and supercompactness, circulated manuscript.

[14] R. Laver, Making the supercompactness of $\kappa$ indestructible under $\kappa$-directed closed forcing, Israel J. Math. 29 (1978), 385-388.

[15] A. Lévy and R. Solovay, Measurable cardinals and the continuum hypothesis, ibid. 5 (1967), 234-248.

[16] M. Magidor, How large is the first strongly compact cardinal?, Ann. Math. Logic 10 (1976), 33-57.

[17] - On the role of supercompact and extendible cardinals in logic, Israel J. Math. 10 (1971), 147-157.

[18] T. Menas, On strong compactness and supercompactness, Ann. Math. Logic 7 (1974), 327-359.

[19] R. Solovay, W. Reinhardt and A. Kanamori, Strong axioms of infinity and elementary embeddings, ibid. 13 (1978), 73-116.

Department of Mathematics

Baruch College of CUNY

New York, NY 10010, U.S.A.

E-mail: awabb@cunyvm.cuny.edu

Web: http://math.baruch.cuny.edu/ apter

Received 14 October 1999;

in revised form 14 September 2000 